

\title{
Autocuidado, adherencia e incertidumbre: tratamientos biomédicos y experiencias de pacientes en el dolor crónico de la migraña
}

\author{
Self-care, adherence and uncertainty: biomedical \\ treatments and patients' experiences regarding \\ chronic migraine pain
}

Del Monaco, Romina ${ }^{1}$

'Licenciada en Sociología, Magíster en Antropología Social y Política. Becaria Doctoral del Consejo Nacional de Investigaciones Científicas y Técnicas (CONICET). Doctoranda en Ciencias Sociales. Docente, Facultad de Ciencias Sociales, Universidad de Buenos Aires. Argentina. rominadelmonaco@yahoo. com.ar
RESUMEN En este escrito se exploran y analizan, desde las ciencias sociales, algunas particularidades de los tratamientos para la migraña desde un enfoque relacional que articula los dichos de los profesionales con las experiencias y prácticas de los pacientes en los servicios de salud y en sus trayectorias de cuidado cotidianas con este dolor. Se realizó una investigación cualitativa a través de entrevistas semiestructuradas a médicos neurólogos y pacientes en un hospital público de la Ciudad Autónoma de Buenos Aires. Se observan un conjunto de procesos por medio de los cuales la biomedicina delega en los pacientes parte de la responsabilidad del curso de su dolencia a través de una lógica de autocuidado. Los sujetos deben realizar numerosos cambios en sus modos de vida a fin de evitar la aparición de los dolores de cabeza. Sin embargo, al no existir certezas en torno a la eficacia de estas modificaciones, la cotidianidad se recubre de incertidumbre y de la exigencia de seguir pautas de cuidado cuyo cumplimiento es valorado no solo por los profesionales sino también socialmente.

PALABRAS CLAVES Dolor Crónico; Transtornos Migrañosos; Tratamiento; Autocuidado; Incertidumbre; Argentina.

\begin{abstract}
Based in the social sciences, this text explores and analyzes the particularities of migraine treatments using a relational approach, articulating the statements of professionals with the experiences and practices of patients in health services and their everyday care trajectories to manage pain. This qualitative research study utilized semi-structured interviews with neurologists and patients in a public hospital in the Autonomous City of Buenos Aires. A number of processes were observed by which biomedicine assigns part of the responsibility for the course of the disease to the patient through the logic of self-care. Patients must make changes in their ways of life in order to prevent headaches from appearing. Nevertheless, as the efficacy of these modifications is unclear, daily life is filled with uncertainty and charged with the mandate to follow care guidelines, which are valued not only professionally but also socially.
\end{abstract}

KEY WORDS Chronic Pain; Migraine Disorders; Therapeutics; Self Care; Uncertainty; Argentina. 


\section{INTRODUCCIÓN}

Las experiencias dolorosas temporarias, ya sean por enfermedad, accidente u otras circunstancias, se consideran "probables" y "normales" en el transcurso de la vida de los sujetos (1). En cambio, los dolores crónicos dejan de ser experiencias temporales y se transforman en una condición que altera los sentidos del padecer y de lograr cierto alivio luego de un tratamiento. De acuerdo a diversos autores, los dolores crónicos (de cabeza, de espalda, etc.) median entre los sujetos y sus relaciones sociales y requieren indagar en los sentidos que adquieren esas vivencias. Además, como son padecimientos que la medicina maneja sin mucha eficacia, los dolores crónicos exponen las contradicciones de la perspectiva médica por tratar al sufrimiento desde técnicas racionales e instrumentales (2). Por ejemplo, los profesionales tienden a asociar la cronicidad con la prolongación en el tiempo y dejan de lado aspectos vinculados a las vidas cotidianas de los pacientes.

Las transformaciones del capitalismo contemporáneo, tales como la desregulación económica, el debilitamiento de las funciones del Estado, la precarización laboral, etc., no solo incrementan la exclusión y la heterogeneidad social (3) sino que influyen en el surgimiento y/o aumento de nuevas formas de padecer. Es decir, hay dimensiones económicas y políticas (locales, nacionales e internacionales) que han disuelto modos de bienestar, producido nuevos vínculos entre el bienestar y el mercado y, por lo tanto, nuevos malestares y padecimientos (4).

El saber biomédico busca las causas de los padecimientos en los cuerpos y, a partir de conocimientos objetivos y científicos, realiza un diagnóstico y propone un tratamiento. De esta forma, la definición de la biomedicina para entender, diagnosticar y tratar ciertos aspectos de las dolencias crónicas, que refieren a los modos de vida de los sujetos, se puede (en parte) explicar por tener como modelo epistemológico de referencia al positivismo. Los cuerpos son objeto de una manipulación atenta, donde se reconstruye un cuerpo "biológico" distinto de aquel con el que interactuamos en la vida cotidiana (5).

Este modelo, que tradicionalmente ha practicado y concebido la medicina, se puede aplicar tanto en aquellas enfermedades que la biomedicina denomina "agudas" caracterizadas, en general, por daños o lesiones orgánicas cuyos tratamientos se orientan a la curación, como en enfermedades crónicas tales como la diabetes, el VIH, la hepatitis $C$, etcétera. En ambos casos existen evidencias fácticas de la existencia de estas enfermedades. Es decir, hay indicadores que mediante estudios (test, análisis, imágenes) dan cuenta de que algo se encuentra fuera de los "parámetros normales" esperados por la medicina. Sin embargo, a diferencia de las enfermedades agudas, las enfermedades crónicas, con distintas significaciones sociales, se definen por tener un desarrollo de largo plazo, ser inciertas en su curso, desarrollo y curación, ser intrusivas, modificar la vida de los pacientes y tener un costo elevado (6).

Por otro lado, existe un conjunto de padecimientos crónicos que, si bien la medicina los diagnostica y trata, se diferencian tanto de las enfermedades denominadas "agudas" como de las crónicas en que no hay evidencias "biológicas" de su realidad fáctica y de sus explicaciones causales, por lo que sus explicaciones tienden a ser difusas y fragmentarias. La migraña es un ejemplo de estos padecimientos crónicos. Se trata de un tipo de dolor de cabeza recurrente, del cual no existen indicadores empíricos que den cuenta de los motivos por los que aparece. Generalmente, estos dolores se dan en la mitad de la cabeza y se acompañan de náuseas, vómitos, molestias e intolerancias a la luz, al ruido y a los olores. Otros síntomas que los acompañan con cierta frecuencia son: molestias en las articulaciones, mareos, sensaciones de cosquilleo y una sensación particular denominada "aura", que según los dichos de los pacientes se trata de alteraciones visuales (destellos de luz, rayos, etc.) y, ocasionalmente, pérdidas temporarias breves de memoria.

Los profesionales entrevistados refieren a la migraña como una enfermedad neurológica, generalmente con base genética. La consideran "un tipo de cefalea primaria" (a), "un dolor de cabeza con particularidades específicas", "una alteración neurológica", etcétera. Las diferentes formas de nombrar un mismo dolor señalan la dificultad biomédica para dar cuenta de un padecimiento que no se corresponde con lo que habitualmente ha entendido la medicina como enfermedad. 
Las características y particularidades de la migraña no solo entran en contradicción con el saber biomédico sino que además permiten discutir con otros estudios que, desde las ciencias sociales, indican que, en los padecimientos crónicos, los profesionales deslegitiman las palabras de los sujetos (2-7). Es decir, a diferencia de otros dolores crónicos, el diagnóstico de la migraña surge de la legitimidad que los médicos le otorgan a los relatos de los sujetos sobre sus experiencias corporales y emocionales con los dolores de cabeza. No solo se escucha y se reconoce lo que dicen los pacientes sino que además la falta de otras evidencias hace que se incorporen y legitimen esas percepciones como partes constitutivas de la definición biomédica del padecimiento. Una vez que la biomedicina quita los rastros de subjetividad y convierte esos dichos en categorías neutrales, objetivas y científicas, los profesionales explican la aparición de los dolores de cabeza combinando indistintamente condiciones biológicas y sociales. Por ejemplo, ser mujer y tener un familiar con migraña pueden ser factores que predisponen al padecimiento, al igual que comer determinados alimentos, tomar alcohol o no realizar actividad física (8).

El saber biomédico legitima la incorporación de las condiciones sociales como posibles causas de los dolores de cabeza mediante la noción epidemiológica de estilo de vida. Es necesario tener una "vida sana" para prevenir futuras enfermedades y/o para mejorar el estado de padecimientos existentes. La biomedicina conceptualiza el cumplimiento de estos aspectos sociales mediante la noción de adherencia. Por adherencia los profesionales entienden el grado de compromiso, comportamientos y actitudes de cumplimiento que los pacientes tienen con los tratamientos (9).

Este tipo de racionalidad y moralidad particular configura un marco valorativo para ciertas conductas y manifestaciones corporales (10). Es decir, la salud se vuelve un concepto central en la identidad moderna que, a través de determinados preceptos provee valores morales al orden cultural e interviene en la constitución de un sí mismo que adquiere valores positivos (cuando es saludable) o negativos (frente a problemas de salud que lo ubican en un lugar de subordinación y estigmatización). El vínculo entre salud y moralidad reside en que el sujeto "saludable" y cierta imagen corporal no solo tiene que ver con cuestiones biológicas sino, principalmente, con un tipo de persona responsable y respetable (11).

La extensión del campo biomédico a la vida cotidiana, que permea distintas prácticas y actividades, es analizado por Foucault a través de la noción de medicalización (12). Conrad (13) profundiza en el estudio de esta categoría y la define como un proceso por el cual problemas no médicos se denominan y tratan como enfermedades o desórdenes, quedando distintos aspectos de la vida cotidiana bajo el dominio, influencia y supervisión de la biomedicina. Según este autor, la medicalización es resultado de procesos socioculturales y su clave reside en que, a partir de la definición de un problema en términos médicos, se pasa a emplear un lenguaje, marco e intervención biomédica para su posterior concepción y tratamiento (13). Se trata de un término que, desde las ciencias sociales, se propone como crítico respecto de la extensión y consecuencias de la biomedicina en distintos ámbitos de la cotidianidad (12-15).

Como resultado de la medicalización de espacios antes aparentemente alejados del saber médico, en este trabajo se indaga y analiza en la lógica de autocuidado (16) como un proceso que no solo modifica las relaciones entre médicos y pacientes a partir de las prácticas y estrategias de cuidado en torno al padecimiento, sino que además incrementa la responsabilidad de los sujetos en relación a su dolencia.

El seguimiento y los resultados de los tratamientos son medidos por los profesionales en función del compromiso y la adherencia de los pacientes respecto de ciertas prácticas de cuidado y cambios en sus modos de vida. Se trata de sujetos activos que surgen de entramados de prácticas y saberes atravesados por dispositivos de poder que regulan modos de cuidado de sí $(12,17)$.

La cotidianidad se encuentra teñida de normas, reglas, regularidades, horarios, alimentos, etc., algunos permitidos, y otros prohibidos y perjudiciales. A su vez, si bien nada garantiza que cumplir con dichas prescripciones influya en la disminución de los dolores de cabeza, la falta de cumplimiento convierte a los sujetos en "pacientes que no adhieren y no se comprometen" cuyas prácticas son sancionadas socialmente por "moralmente incorrectas". 


\section{INVESTIGACIÓN Y MÉTODOS}

La investigación adoptó los lineamientos de la metodología cualitativa. Se describen y analizan los relatos de profesionales y pacientes (b) sobre determinadas características del proceso de tratamiento de la migraña. Este abordaje permite tomar contacto con el punto de vista de los actores a través de sus propias narrativas, y posibilita acceder a aspectos subjetivos y simbólicos de la población que se estudia (18). El trabajo de campo se desarrolló durante el año 2010 y el primer cuatrimestre de 2011, en el servicio de neurología de un hospital público de la Ciudad Autónoma de Buenos Aires. Se realizaron 38 entrevistas semiestructuradas a pacientes con migraña (29 mujeres y 9 varones) de sector socioeconómico medio/medio bajo. El rango etario iba desde los 21 hasta los 60 años.

Se entrevistaron a 15 profesionales en el mismo hospital público (8 mujeres y 7 varones). De las mujeres entrevistadas el rango etario iba desde los 35 hasta los 60 años y, en el caso de los varones, desde los 34 hasta los 70 años. Respecto del desempeño profesional cabe aclarar que tres entrevistados/as eran neurólogos/as especialistas en el tratamiento de la migraña. Luego, se entrevistó a 7 médicos/as neurólogos/as en general (especialistas en Parkinson, movimientos anormales, neurología general, etc.). Por último, se entrevistó a 5 médicos/as residentes del último año del servicio de neurología. También, dados los antecedentes que tenían en los tratamientos para la migraña, se entrevistó a dos profesionales neurólogos (una mujer de 45 años y un varón de 57 años) que trabajaban en una clínica privada.

Tanto en el caso de los médicos como de los pacientes, se utilizó una guía que se complementó e intercaló con preguntas espontáneas surgidas en el contexto de las entrevistas, cuyo proceso de diseño estuvo guiado por un conjunto de categorías que, acorde a las investigaciones previas, han probado ser de importancia en los estudios sobre padecimientos crónicos. Con el avance del trabajo de campo, este sistema de categorías fue revisado, ampliado y modificado.

La teoría fundamentada guió la construcción de teoría basada en datos empíricos siguiendo un proceso de análisis inductivo. El número de entrevistas y de observaciones realizadas está dado por el criterio de saturación, es decir, momento de la investigación en que se deja de obtener información nueva respecto de las categorías centrales de acuerdo a la relevancia teórica de los datos (19).

\section{Análisis de la información}

Las entrevistas fueron codificadas y analizadas sobre la base de categorías temáticas que guiaron el proceso de análisis de los datos:

- Entrevistas a pacientes: condiciones generales de vida, experiencias en relación con el padecimiento, modificaciones en las vidas cotidianas a partir de padecimiento, trayectorias y características de los pacientes en las prácticas de cuidado para la migraña.

- Entrevistas a profesionales: caracterización del padecimiento y diagnóstico, prácticas terapéuticas (tipos de tratamiento, formas de prevención médicas y/o hábitos y costumbres que según los profesionales los pacientes pueden adquirir para disminuir la frecuencia de los dolores de cabeza).

Asimismo, se realizó observación participante en la sala de espera y en ateneos a residentes del área de neurología. En primer lugar, fue posible presenciar una serie de ateneos donde profesionales especialistas en migraña presentaban ante los residentes las particularidades de este tipo de dolores de cabeza (distintas teorías sobre su origen, etapas necesarias de las consultas, modos de prevención, etcétera.). Dichas observaciones posibilitaron conocer las dudas y las preguntas que los residentes les hacían a los profesionales sobre este diagnóstico, así como también la caracterización que dentro de la medicina se realiza de esta dolencia.

La sala de espera para los sujetos que se atendían por migraña era compartida con pacientes que concurrían al hospital por diferentes problemas neurológicos y se registraron diálogos, conversaciones informales y comentarios sobre las diversas dolencias. En ocasiones, fue posible dialogar con sujetos entrevistados anteriormente, que concurrían al hospital para un seguimiento de su tratamiento. Estos diálogos permitieron conocer los cambios y las consecuencias durante los 
tratamientos, así como diversas cuestiones de la cotidianidad con los dolores de cabeza que no se habían mencionado en las entrevistas previas.

\section{Resguardos éticos}

Esta investigación se adecuó a los criterios de consentimiento informado y confidencialidad que se aplican en los estudios sobre salud, con el fin de asegurar los derechos de los/as participantes, así como también de resguardar su identidad. Los sujetos entrevistados son mayores de 18 años. Para realizar el trabajo de campo en el servicio de salud, se llevó a cabo el proceso de evaluación requerido a través del comité de ética del hospital.

\section{RESULTADOS}

\section{Cuidado, adherencia e incertidumbre}

Digamos que hay una cuestión genética que predispone biológicamente. A eso, de nuevo, como todo en la medicina, le tenés que sumar los factores del medio. Yo puedo tener en mi familia predisposición a que haya antecedentes genéticos de problemas oncológicos, pero también el tipo de vida que llevo hace que eso se manifieste o no. Con la migraña pasa lo mismo. Si yo, además de tener familiares con migraña, duermo mal, como mal, me gusta tomar vino seguido y demás, y... mis episodios de migraña van a ser cada vez más seguidos. (Médica neuróloga)

La práctica médica transforma los padecimientos en patologías, es decir, en hechos objetivos basados en evidencias empíricas estructuradas en el correspondiente campo biológico. En general, de esas observaciones se desprende un diagnóstico preciso, un tratamiento y, en algunos casos, una cura. Este modelo de enfermedad asociado a daños y/o lesiones orgánicas promueve la curación a través de la realización de tratamientos. Además, los profesionales construyen la enfermedad y el cuerpo de los pacientes como objetos médicos (5). Se trata de discursos de carácter generalizante y validez universal (20). Es decir, la resolución de ciertas enfermedades (en especial aquellas que la medicina denomina "agudas") surge de tratamientos estandarizados cuya aplicación tiende a ser homogénea independientemente de los sujetos que padecen.

No obstante, el saber biomédico se ve cuestionado por el aumento de padecimientos crónicos como la migraña que no responden al modelo etiológico normatizado. Por eso, con el tiempo, a la lógica curativa mencionada previamente, se le agregó una muy distinta cuyo objetivo deja de ser la curación. Son tratamientos dirigidos a disminuir (o evitar que empeoren) los síntomas de padecimientos crónicos.

Por el momento, de acuerdo a los relatos de los profesionales, la migraña no tiene cura. El objetivo de los tratamientos es reducir la frecuencia e intensidad de los dolores de cabeza y de los demás síntomas con el fin de mejorar la calidad de vida de quienes padecen.

El conocimiento de la imposibilidad de curación, tanto de médicos como pacientes, interviene en los relatos de los sujetos respecto de los modos de actuar cuando aparece el dolor y en sus percepciones sobre este padecimiento y sobre los tratamientos. Por ejemplo, ante la aparición (inesperada y repentina) de la migraña, existe una rutina y un conjunto de prácticas que se caracterizan, entre otras cosas, por aislarse, apagar luces, quedarse quietos, etcétera. En relación con los tratamientos, se observa por momentos una mezcla de acostumbramiento y resignación, saber que la migraña no tiene cura disminuye las expectativas respecto de los resultados pero, al mismo tiempo, incrementa la sensación de angustia el hecho de no saber qué hacer para disminuir su frecuencia e intensidad. El conocimiento de la imposibilidad de curación también interviene en las trayectorias de los sujetos en los servicios de salud y en las distintas búsquedas de respuestas a estos dolores.

Se trata de técnicas y procedimientos que son variables, numerosos y se transforman de acuerdo a las condiciones socioeconómicas de los sujetos y al contexto histórico. A su vez, la cronicidad modifica la percepción del dolor como algo externo y extraño. Por momentos, y con el tiempo, se convierte en algo con lo que quienes padecen dicen "aprender a convivir". 
Según el saber biomédico, en las últimas décadas se asiste a una complejización de los factores involucrados en el desarrollo de ciertas enfermedades. Existen variables psicosociales (sistematizadas como estilo de vida) que influyen en el desarrollo de dolencias, y por eso es necesario controlarlas mediante prevención y tratamientos (21). En este sentido, diferentes perspectivas teóricas, el autocuidado (desde las ciencias sociales) y el estilo de vida (desde la biomedicina), refieren a cómo los sujetos, progresivamente, deben hacerse cargo del cuidado de su enfermedad a través de controles y cambios en sus modos de vida.

En los relatos de los médicos "se combina lo farmacológico con lo no farmacológico", es decir, medicamentos con cambios en las prácticas y comportamientos cotidianos. La mayoría de los neurólogos entrevistados dijeron que los estilos de vida son una posible explicación causal del origen de la migraña, y también un factor a tener en cuenta para prevenir y para tratar, una vez que se tiene la enfermedad, la aparición de los síntomas o dolores de cabeza. Esta noción genera una especie de círculo vicioso a través del cual los sujetos deben seguir un conjunto de pautas y se convierten en responsables, no solo de la aparición de los síntomas sino también -en aquellos casos que no tienen antecedentes biológicos (por ejemplo, familiares con migraña)- del éxito o no de los tratamientos y de la aparición de la enfermedad.

Al tiempo que los profesionales informan las conductas adecuadas para prevenir los dolores de cabeza, delegan en los sujetos la responsabilidad de controlar y estar atentos sobre su propio cuerpo y modificar prácticas y comportamientos. Las indicaciones médicas encubiertas por el concepto de autocuidado, son vividas por los sujetos como prescripciones, que revisten la forma de un mandato que implica una lógica extraña a su cotidianidad (16 p.91)

Las estrategias de autocuidado evidencian los modos en que la práctica médica ya no concentra su poder solo en la institución, sino que ejerce influencia en otros ámbitos y espacios de la vida relacionados con condiciones íntimas y personales de los pacientes. Es decir, numerosas prácticas y modos de vida son sistematizados e incorporados como indicadores que pueden mejorar, prevenir, controlar o impedir ciertas enfermedades.
Por eso, el complejo de autocuidado permite explorar y analizar cómo las relaciones entre médicos y pacientes se cubren y tensionan de exigencias de compromiso y adherencia en las cuales los sujetos se convierten en responsables de su dolencia frente a las demandas de la biomedicina.

Los procesos de medicalización de las vidas cotidianas (12) extienden el campo de la medicina a otros contextos y permean actividades y acciones de los sujetos modificando las relaciones consigo mismos y con los otros. Según los profesionales entrevistados, cumplir con estas pautas no es una tarea sencilla, y por eso encuentran dificultades para que los sujetos le den importancia a la dolencia y adhieran a los tratamientos. También reconocieron que "una persona con migraña puede pasar diez años con el dolor y no consultar con nadie" (Médico neurólogo). La adherencia es una categoría médica que implica una actitud de cumplimiento o incumplimiento de los tratamientos a partir de las acciones que los sujetos realizan (o no) para mejorar su salud. En esta definición está implícito que los comportamientos cotidianos de los pacientes se evalúan desde el punto de vista de los profesionales (9).

En cambio, para los sujetos, "hacerse cargo de la enfermedad" y cumplir con los tratamientos y los medicamentos prescriptos por los profesionales no depende solamente de conocer cuáles son los desencadenantes de la migraña. Existen condiciones sociales y económicas que influyen en el acceso a medicamentos, servicios de salud, cambios en los hábitos alimentarios, realización de actividad física, etcétera. Incluso, en muchos casos dijeron que, a pesar de controlar la variedad de cuestiones prescriptas por los profesionales, los dolores vuelven.

Ser saludable pasa a estar ligado a cuestiones morales, al control de sí y a la buena presencia. Se trata de una noción cuyo dominio simbólico crea y recrea al sí mismo en relación a un "sí mismo social", es decir, una moralidad dominante que guía pautas aceptadas o no (21). Combinar las narrativas de los profesionales con las de los pacientes posibilita examinar los modos en que, la responsabilidad que reclaman los médicos se transforma, por momentos, en responsabilización y culpabilización de los sujetos. 


\section{Idas y venidas: sobre la continuidad en los tratamientos}

\begin{abstract}
La migraña es algo tan frecuente que la mayoría de la gente no la respeta como cuadro de enfermedad. No lo respeta la gente y no lo respetan los propios pacientes. Porque, por ejemplo, nadie se queda con un dedo que se le empieza a poner negro un día, dos días, tres días, van al médico enseguida. Con un dolor de pecho también. Acá nosotros vemos que hay gente que hace diez años que todos los días tienen dolor de cabeza. Porque me tenía que acostumbrar a vivir así, te dicen, a este dolor no lo respetan. (Médico neurólogo)
\end{abstract}

La espera o demora en consultar a los servicios de salud por los dolores de cabeza es algo que repitieron tanto los médicos como los pacientes. Para los profesionales "las personas no respetan el dolor y por eso dejan pasar tanto tiempo, en otras enfermedades eso no pasa" (Médico neurólogo).

La mayoría de los sujetos con migraña dijeron tener dolores de cabeza desde hace varios años. No obstante, las razones por las cuales "se aguantan" difieren de los relatos de los médicos. Es decir, la técnica corporal del aguante no solo modifica los patrones del sentir, sus modos de expresión y la valoración social, sino que produce, reproduce y responde a ciertos mandatos y acusaciones de discursos oficiales y/o dominantes (4 p.232). Las personas con migraña contaron que uno de los motivos por los cuales tenían que "aguantar y seguir" se relaciona con las características sociales atribuidas a los dolores de cabeza que los vinculan con excusas y mentiras. Es decir, hay un conjunto de discursos (biomédicos, sociales) que influyen y condicionan a que, en algunos padecimientos, haya que aguantar y en otros no.

En algunos casos dicen "fui de un neurólogo al otro pero sin solución y entonces después no fui más porque la verdad era siempre lo mismo, que te hacemos el electro y que el electro sale bien..." (Susana, 39 años).

Te digo, después de hacer muchos tratamientos, es como que tampoco quería venir acá, porque ya estás como desanimado... No vine enseguida porque no quiero ir a uno y que falle, y a otro y que falle, y a otro.
Obviamente que querés la solución, pero estás un poco desanimado porque incluso tuve efectos colaterales por los remedios, como aumentar mucho de peso y nunca bajé esos kilos, entonces puse esfuerzos y seguía con dolor de cabeza. (Eric, 47 años)

Antes de la consulta con el neurólogo actual, los pacientes dicen tener una trayectoria por distintos profesionales signada por la desilusión de tratamientos que no habían funcionado. Otros, relataron convivir con los dolores de cabeza desde hacía años pero aclararon: "para qué iba a consultar, si el dolor de cabeza es algo común" (Lucía, 35 años). A partir de las palabras de los sujetos sobre las percepciones que tienen del entorno en relación con los dolores de cabeza, se puede inferir que se trata de un padecimiento vinculado con lo común y frecuente ya que "hay cosas más importantes por las que ir al médico ¿no?" (Lucía, 35 años).

Los motivos que fomentan las demoras en las consultas son múltiples y variados. Las condiciones económicas y sociales de los pacientes son un aspecto que influye significativamente. Primero, en lo que respecta a las consultas, se requiere acceder a la información de que en determinados servicios de salud existen gabinetes especializados en el tratamiento de la migraña. En segundo lugar, se necesita disponer del tiempo para concurrir, por ejemplo, un día de semana por la mañana a las consultas. En caso de acceder y de comenzar un tratamiento, la mayoría de los pacientes manifestó que una de las dificultades era el costo de los medicamentos recetados por los profesionales, ya que muchos de ellos son importados.

\footnotetext{
$Y$ para mi presupuesto se complica, no cuestan quinientos mangos pero... setenta, ochenta pesos y, si no tengo recetarios de la obra social, que a veces puedo tener... me lo tengo que comprar sin descuento. Ella me da Ketorolac para tomar no bien me empieza el dolor, que es bastante caro, entonces yo le comenté si lo puedo manejar con Ibuprofeno, que es más barato, y me dijo que estaba bien. (Mirtha, 41 años)
}

Los problemas económicos que relata Mirtha para acceder a los medicamentos para la migraña, 
son frecuentes en otros entrevistados y ponen de manifiesto los límites ante los que se encuentran algunos pacientes para poder mantener los tratamientos en el tiempo. Otro de los motivos que pueden fomentar las demoras en las consultas o el abandono de los tratamientos es el saber generalizado (tanto en los dichos de los médicos como de pacientes) sobre las limitaciones en la eficacia de los tratamientos para la migraña. En palabras de uno de los profesionales: "la migraña tiene un origen biológico, pero todavía no se pudo aislar el gen" (Médica neuróloga). Son tantas las condiciones que tienen que tener en cuenta los sujetos para evitar los dolores de cabeza que, a modo de interrogante, cabe preguntarse: ¿qué posibilidades existen de que alguien con migraña cumpla con todos los requisitos propuestos por el saber biomédico? Es decir, alimentación, actividad física, horas de sueño, herencia, etcétera.

Lo que pasa es que yo me mandé varias macanas de cortar el tratamiento. Por esto mismo que te decía, que no quería tomar muchas pastillas. A lo que me dice la doctora que si seguimos un tratamiento es difícil bajar la dosis cuando yo no lo sigo. Porque yo me tomé el atrevimiento el año pasado de sentirme bien y entonces dije: bueno la dejo de tomar. Ella me dice que si hago bien el tratamiento se va a ir bajando la dosis y no voy a tomar más. (María, 26 años)

Algunas personas, como María, mencionaron que

...en épocas en las que estoy peor vuelvo, pero si veo una mejora dejo un tiempo de venir. (Lautaro, 30 años)

...cuando me canso de tanto dolor de cabeza me decido a consultar. (Bárbara, 27 años)

Si bien en este trabajo se focaliza en las relaciones y tensiones entre profesionales y pacientes a partir del análisis de las características de los tratamientos para la migraña, mediante las narrativas de los sujetos fue posible rastrear distintas percepciones y estrategias de cuidado alternativas o complementarias a los tratamientos biomédicos.

Las búsquedas de otros tipos de tratamientos alternativos de la biomedicina para la migraña, por parte de los pacientes, fueron variadas y disímiles.
En algunos casos, los sujetos mencionan que si bien no probaron otro tipo de medicina:

...tengo ganas porque la medicina holística potencia lo mínimo de medicamentos y está más relacionada con lo natural. (Edgardo, 38 años)

...sabés que no, pero me gustaría ver... una de las abuelas con las que trabajo me dijo que ella tomaba unas gotitas a la mañana... no sé para qué será... ella me dice ¿por qué no vas a otro médico? (Julia, 58 años)

Asimismo, hay sujetos que dicen haber probado otras opciones y relatan sus experiencias con diversos tratamientos (homeopatía, acupuntura, etcétera):

\begin{abstract}
...me traté con un homeópata casi dos años pero no me dio resultado... después hice acupuntura... dos años y medio pero como había dejado el migral no me dio resultado porque no me gusta mezclar. (Silvia, 47 años)
\end{abstract}

También, es frecuente que los sujetos refieran a una combinación entre los tratamientos que dan los profesionales de la salud y aquellos con lo que "los doctores no están muy de acuerdo". Este tipo de estrategias incluye terapia, yuyos, tés, etcétera:

Probé de todo porque encima tengo una amiga que también tiene cefalea entonces siempre estamos con los yuyos... o sea, té de tilo, té de manzanilla... bueno, que a los médicos mucho como que no le gusta. (Patricia, 26 años)

Te digo que fui hasta a Umbanda, yo me acuerdo que me hizo agachar y me lavó la cabeza con cerveza... nunca más... después ya te digo, los que tiran las cartas, a la Iglesia Universal del Reino de Dios... a todo lo que vos te puedas imaginar. Y cuando dicen "es falta de inteligencia que la gente va a esos lugares" yo digo que es la desesperación, de no saber qué hacer. (Mirtha, 54 años)

La posibilidad de ir y venir en los tratamientos sin temor a empeorar o morirse tiene que ver con 
una particularidad de la migraña que un profesional definió de la siguiente manera: "la migraña tiene algo bueno y algo malo, lo malo es que no se cura y lo bueno es que no te vas a morir por los dolores de cabeza" (Médico neurólogo).

La mayoría de los médicos acuerdan en que existen posibilidades de que los tratamientos farmacológicos no funcionen y que haya que cambiar los medicamentos por otros. Sin embargo, no ponen en duda que los cambios en los estilos de vida, es decir, las modificaciones de los hábitos y acciones de los pacientes sean un elemento fundamental para prevenir la aparición de los dolores de cabeza.

\section{Cronicidad, compromiso y autocuidado}

Esto dista mucho de la pastilla mágica que vino a buscar el paciente, el compromiso es fundamental. (Médico neurólogo)

En los últimos tiempos, el aumento de los dolores crónicos modificó las formas que tiene la práctica biomédica de nombrar y tratar ciertas enfermedades. Pueden existir múltiples condiciones que se superponen e intervienen en los padecimientos. También, en lugar de hablar de curación, los médicos hacen referencia a "procesos", "mantenimiento", "cuidado".

Respecto de los tratamientos farmacológicos, consisten en medicaciones prescriptas por los profesionales y pueden ser de dos clases. Por un lado, medicación preventiva, que "generalmente se usa mucho en las migrañas, vos indicás una medicación, para evitar la recurrencia del dolor de cabeza y lo tiene que tomar diariamente" (Médica neuróloga). Por otro lado, los profesionales denominan medicación abortiva, de ataque o rescate a las pastillas que se toman no bien empiezan los dolores de cabeza: "el abortivo es el que hacemos todos, me duele la cabeza y me tomo un analgésico" (Médica neuróloga).

Sin embargo, como se mencionó previamente, tanto en las teorías sobre el surgimiento de la migraña como en las explicaciones sobre los modos de prevención, los profesionales le otorgan un papel protagónico a las acciones y prácticas de los sujetos. Son reiterativas las menciones a que "con la medicación sola no, porque el paciente migrañoso es muy sensible y tendría que acostarse a la misma hora, comer seguido, tomar mucha agua, hacer actividad física" (Médico neurólogo). A su vez, en los relatos médicos no son frecuentes las referencias a los problemas y las dificultades que pueden intervenir e interrumpir los tratamientos, tales como falta de tiempo, dinero, información para acceder a las consultas, complicaciones para comer ciertos alimentos, etcétera. Se convierte a las prácticas terapéuticas en actividades y cambios cotidianos de por vida, que dejan de lado las condiciones que hacen que existan diferentes formas de sobrellevar un mismo padecimiento.

Serían como medidas preventivas para evitar que aparezca; en realidad son los hábitos diarios, indicamos para la migraña hacer ejercicios de relajación, tai chi, yoga, pilates, ahora que está de moda. Después se les recomienda no ayunar, o sea ser regular y ordenado con la alimentación, se les recomienda un buen sueño, o sea mínimo las siete u ocho horas diarias, buena hidratación, evitar el café en lo posible. Por ahí el tabaco se les puede decir, y después hay un montón de medidas higiénicas, dietéticas que también están descriptas, pero uno las va condicionando de acuerdo a lo que te comenta el paciente. (Médica neuróloga)

La cronicidad instala una nueva forma de tratar los padecimientos que combina y complementa lo farmacológico con medidas higiénicodietéticas (por ejemplo, evitar el exceso de peso, la falta de sueño, el sedentarismo, la falta de ejercicio y "aprender" a controlar los desencadenantes del dolor, diferentes según los sujetos).

Si bien la lógica de autocuidado aparenta una mayor autonomía de los pacientes debido a una extensión de los espacios de decisión respecto al padecimiento, al mismo tiempo, si los dolores persisten, se trata de una lógica que convierte a los sujetos en responsables de su situación. Es decir, si la migraña continúa con igual frecuencia e intensidad que antes de los tratamientos, surgen distintas teorías que, dependiendo de los actores, explican por qué persisten los dolores de cabeza.

Los profesionales plantean un sujeto que, desde una visión individualista y racional, elige seguir determinadas pautas en pos de su salud. Al mismo tiempo, refieren a la falta de compromiso de los pacientes a 
los tratamientos. Señalan que "en la migraña no se adhiere como en otras enfermedades, es cíclica, cuando hay mejoras se deja de concurrir y cuando vuelve el dolor se vuelve" (Médica neuróloga).

Lo que sí me parece, es que lo que uno le tiene que hacer entender a los pacientes es que se tienen que comprometer. Y creo que es una de las cosas más difíciles de lograr. La atención al paciente con dolor crónico es el compromiso, es el involucramiento, la aceptación [...] Decir, bueno: "Usted es el responsable de esto, de llegar a buen puerto, no de los médicos". Y es difícil hacerles entender esto a los pacientes. Por eso los pacientes buscan alguna cosa mágica y van peregrinando de médico en médico, que lo único que hacen es cambiarles la pastilla. Por eso también la adhesión al tratamiento es muy variable, en general los pacientes no adhieren como en otras enfermedades neurológicas. (Médico neurólogo)

La categoría biomédica de adherencia se construye sobre un modelo normativo, es decir, del "deber ser" caracterizado por una profunda responsabilidad de los sujetos frente a su enfermedad y resolución. Así, se plantea una tipología discursiva que clasifica a los pacientes en buenos y malos, es decir, en pacientes adherentes o potencialmente adherentes y en pacientes no adherentes (9 p.289). No obstante, el estudio de dolencias crónicas requiere superar la visión de un individuo que no se compromete o no adhiere e indagar en las relaciones entre los dolores y las experiencias y trayectorias personales (unido a los sentidos sociales atribuidos a los dolores de cabeza).

Es que para la gente es más fácil las cosas que menos lo comprometen. Porque salir a caminar, bajarte antes de la parada del colectivo... Qué sé yo, no sé, organizarte con la comida, comer mejor... Si uno ataca esa cuestión que es más barata, digamos, vas a tener más resultado, tendrías menos dolor. Digamos que es mucho más sacrificado entre comillas. Porque requiere... te ponen la pelota en tu campo, ¿viste? Es muy cómodo que el médico te dé... te lo solucione. El tema es cuando vos tenés la pelota en tu campo y la tenés que manejar vos. Ese es el tema. (Médico neurólogo)
Para la mayoría de los profesionales entrevistados, la adherencia es una actitud y conducta fundamental en el proceso de gestión de la enfermedad que sobrepasa al mundo biomédico porque se extiende a otras esferas de la vida cotidiana por períodos de tiempo indeterminados (22). Se trata de cambios de vida de los sujetos que incluyen "tener paciencia y no querer poner el techo antes que las paredes" (Médico neurólogo).

A través del tratamiento los pacientes no solo deben seguir una disciplina y control que les ayude a disminuir sus dolores de cabeza sino que también, este cumplimiento les posibilita tener una imagen social moralmente aceptable donde los demás reconocen el compromiso, preocupación e interés por el cuidado de su salud.

\section{Conocimientos, responsabilidad e incertidumbre}

Veo qué puedo hacer para evitar que me duela la cabeza. Hago caso, tomo mucho líquido que me evita tener dolores de cabeza; dejar de fumar me separó bastante las migrañas fuertes. Eh... el estrés, el dormir poco, no comer en horario, esas son cosas que me provocan seguro una migraña a la tarde. O sea que necesito como tener un orden, necesito tener una vida medianamente ordenada si quiero que mi día termine feliz. (Carla, 38 años)

El proceso biomédico de recuperar y legitimar los dichos de los pacientes sobre las características de sus dolores de cabeza forma parte de una lógica que les permite a los profesionales dar respuestas a enfermedades que no encuadran en las características de su modelo epistemológico. Los médicos agrupan un conjunto heterogéneo de relatos sobre síntomas y sensaciones y los convierten en una enfermedad delimitada y re-conocida.

Asimismo, les piden a los sujetos que lleven un diario sobre la migraña para conocer sus estilos de vida, en los que los pacientes dicen anotar la cantidad de veces que les duele la cabeza hasta la próxima consulta, los momentos del día en que aparecen los dolores y a qué situaciones vinculan esta aparición. Para los profesionales, se trata de 
...un registro de su vida cotidiana y las cosas más comunes que no se pueden dejar de preguntar son: los alimentos que consumen, horas de sueño, si realizan actividad física, etc., así uno puede ir detectando y sacando lo que está perjudicando a la migraña. (Médico neurólogo)

Son formas de cuidado, prácticas, estrategias que los sujetos con migraña deben llevar a cabo para conocer los posibles desencadenantes del dolor y prevenir su aparición. En algunos casos pueden funcionar $y$, en otros, no. Por eso, se trata de conocimientos fragmentados sobre posibles modos de cuidarse y convivir con este padecimiento.

Desde las ciencias sociales, diversos autores señalan las dificultades que encuentran los pacientes crónicos para que los profesionales biomédicos "crean" en su dolor. Según esta perspectiva, para la biomedicina aquello de lo que se queja un paciente es significativo si refleja un estado fisiológico y, si no se detecta tal referente empírico el sentido de la queja se pone en tela de juicio (5 p.35). No obstante, el dolor crónico de la migraña contradice estos supuestos y demuestra la relevancia e importancia que le dan los profesionales de la salud a los relatos de quienes padecen para la construcción biomédica de la enfermedad.

Dentro de mis posibilidades uno hace lo que puede, vos tenés una enfermedad que tenés que hacer una dieta estricta, no tenés que comer esto, tenés que dormir ocho horas por día y qué se yo... no se puede [...] Vivo para la migraña porque tiene que ser muy responsable un paciente con migraña en ese sentido, es una rutina muy rigurosa y además hay que tener ganas, a lo mejor uno está cansado, viene de trabajar y querés disfrutar con tu familia y no, tengo que hacer natación porque si no, me agarra migraña. (Edgardo, 38 años)

La afirmación de Edgardo respecto de "vivo para la migraña" manifiesta con claridad y logra resumir cuán invasiva puede ser la migraña en la cotidianidad. "Vivir para" implica un alto grado de incertidumbre (si existieran algunas certezas en torno a la aparición del dolor y alivio, la posibilidad de "relajarse" aumentaría). También significa sensaciones de temor a que en cualquier momento aparezca el dolor, a no saber qué hacer para prevenirlo y a la imposibilidad de cumplir con todas las prescripciones médicas en torno a los cambios en el "estilo de vida" (alimentos, actividad física, horas de sueño, estrés, etc.). A su vez, no cumplir con esas prescripciones tiende a convertir a los sujetos en pacientes que no se comprometen, que no les importa su bienestar.

El autocontrol como eje terapéutico y de transmisión de una normalidad, supone como alternativa opuesta y no explicitada el des-control, referido a una actitud del sujeto respecto a su modo de vida, signada por la ausencia de un ajuste de las conductas propias (15). De esta forma, el autocuidado se convierte en un sistema de atención-cuidado-control de sí donde el sujeto adopta una permanente atención sobre ciertos aspectos de su cuerpo y modo de vida que entran en contradicción con los modos previos de construir la experiencia (16). Es decir, en el padecimiento de la migraña, la preocupación por evitar desarreglos y desórdenes en la cotidianidad convierte a los sujetos en personas atentas y preocupadas por cuestiones aparentemente (para aquellos que no tienen estos dolores) insignificantes que intervienen y modifican sus relaciones vinculares e interacciones diarias.

\section{REFLEXIONES FINALES}

En las sociedades actuales los sujetos "saludables" no solo refieren a la ausencia de enfermedad sino también a personas sanas $y$ equilibradas. El sí mismo saludable se sostiene, además, por la creación de otros no saludables categorizados o imaginados como aquellos que corporizan todas las cualidades que quedan por fuera de lo que se debe hacer para estar sano (11).

Estas exigencias a los sujetos contemporáneos parecen entrar en contradicción con padecimiento crónicos como la migraña. En primer lugar, se trata de un dolor que se repite en el tiempo, no se sabe cuánto dura y modifica la vida cotidiana de quien lo padece. A su vez, la migraña presenta ciertas características (en torno a su surgimiento y formas de cuidado) que, en este escrito, permitieron explorar y analizar las relaciones (y tensiones) entre profesionales de la salud 
y pacientes. Tanto las explicaciones sobre los motivos por los cuales aparece este dolor como las formas de tratarlo reposan, principalmente, en un conjunto de indicadores sociales que conforman el estilo de vida del paciente. De esta forma, para prevenir y tratar los dolores de cabeza los profesionales incluyen, como parte fundamental de los tratamientos, cambios significativos en los modos de vida que intervienen en sus experiencias corporales y emocionales. Entonces, los pacientes aprenden a agudizar su atención y preocupación por eventos y situaciones, aparentemente sin sentido para quienes no tienen migraña, pero que en ellos pueden desencadenar dolores de cabeza.

Los límites entre actividades saludables y no saludables se transforman en límites entre sujetos preocupados o no por su salud. Si bien los diagnósticos y tratamientos médicos buscan apoyarse en fundamentos racionales, en el caso de la migraña, los modos de evaluar y tratar al padecimiento incorporan cuestiones sociales, morales y culturales que van más allá de la descripción biológica de un cuerpo. Se trata de un padecimiento que trasciende lo orgánico e indica categorías morales que enfrentan a los sujetos con estos dolores con una dieta particular, preocupación por el cuidado personal y la higiene, etcétera.

El autocuidado, como disciplina y autocontrol, se ha convertido en un modelo dominante de cuidado corporal. De acuerdo a este modelo, el cuerpo saludable representa moralidad, responsabilidad y bienestar (dimensiones centrales de la identidad en las sociedades dominantes) (23). Una persona que se preocupa por su salud y realiza prácticas saludables es estimada y valorada socialmente. En cambio, cuando los dolores de cabeza permanecen, el sujeto tiende a convertirse en responsable de dicha situación por su falta de adherencia y compromiso al tratamiento biomédico.

Lo paradójico de este tipo de dolores de cabeza es que las prácticas de autocuidado incluyen innumerables modificaciones que generan en los sujetos que padecen la sensación de que "siempre hay algo que se escapa". Sus relatos sobre la convivencia con el dolor y las trayectorias con los tratamientos están plagados de expresiones tales como: "incertidumbre", "angustia", "falta de certezas", "fracaso".

En el caso de los profesionales, si bien reconocen que no hay certezas ni forma de garantizar que determinado tratamiento reduzca la aparición de la migraña, resaltaron que para lograr mejoras y una "buena evolución de los tratamientos" es necesario de parte de los sujetos: "compromiso", "responsabilidad", "adherencia" y, sobre todo, ser un "paciente ordenado".

Vos tratás de llevar una vida tranquila, dentro de lo posible, pero no hay garantía de nada, en algún momento intenté buscar certezas en el tratamiento pero tenés que acostumbrarte, certezas no hay. (Eduardo, 38 años)

Te soy sincero, yo algo que relacioné fue el alcohol, así que lo dejé completamente, con tal de vivir mejor. Igual, lo peor es que cuando no tenés dolor y estás con el temor de que algo te va a hacer mal y te va a aparecer... porque hay, como decía la doctora y yo coincido, algunas cosas que salen sin razón. (Eric, 47 años)

Tanto Eric como Eduardo coinciden en la imposibilidad de certezas sobre la migraña. La vida cotidiana se convierte en un espacio minado de posibles detonantes para el dolor que hay que vigilar y cuidar. Mientras que el proceso de medicalización más evidente supone una concentración del poder y saber en la institución médica, el complejo de autocuidado supone un descentramiento de este lugar y una multiplicación de los nudos y focos de la red medicalizadora. Tanto el cuerpo como las relaciones sociales se ven sometidos a una mirada normatizadora que observa, evalúa y compara en relación con los patrones ideales sostenidos desde el conocimiento científico dominante, sin dejar espacio que no sea invadido y contaminado por la "enfermedad" (16 p.93). 


\section{NOTAS FINALES}

a. La noción de cefalea es utilizada en reiteradas oportunidades por los profesionales entrevistados para referir a la migraña y significa, según un médico neurólogo, "una experiencia de dolor craneana, es decir es como el nombre técnico, como la familia de todos. Dentro de las cefaleas hay algunas que son primarias y otras secundarias. Las primarias son aquellas dentro de las cuales los estudios no muestran alteraciones. Las secundarias están relacionadas con una situación en la cual vos tratás el problema que generó el dolor y el dolor se resuelve. Dentro de la cefalea primaria está la migraña que viene de hemicránea y significa mitad de cabeza, porque duele la mitad".

b. Si bien en este trabajo se define una noción de sujeto, dado que la investigación se realizó en un sistema hospitalario, se va a hablar de manera indiferente de paciente y sujeto.

\section{AGRADECIMIENTOS}

Este trabajo es resultado de una investigación mayor en curso denominada "Experiencias, corporalidad y saberes sobre la migraña: un estudio sobre las relaciones entre procesos sociales y vida cotidiana en este dolor crónico en el área metropolitana de Buenos Aires". Agradezco al Consejo Nacional de Investigaciones Científicas y Técnicas (CONICET) que a través de una beca doctoral financia dicha investigación, y agradezco especialmente a la Dra. María Epele por su dedicación y generosidad en la dirección.

\section{REFERENCIAS BIBLIOGRÁFICAS}

1. Hilbert R. The acultural dimension of chronic pain: flawed reality construction and the problem of meaning. Berkeley: University of California Press; 1984.

2. Good B. Pain as human experience: An anthropological perspective. Berkeley: University of California Press; 1994.

3. Svampa M. La sociedad excluyente: Argentina bajo el signo del neoliberalismo. Buenos Aires: Taurus; 2005.

4. Epele M. Sujetar por la herida: Una etnografía sobre drogas, pobreza y salud. Buenos Aires: Paidós; 2010.

5. Good BJ. Medicine, rationality and experience: An anthropological perspective. Cambridge: Cambridge University Press; 1994.

6. Strauss AL, Corbin J, Fagerhaugh S, Glaser BG, Maines D, Suczek B, et al. Chronic illness and the quality of life. 2a ed. St Louis: Mosby; 1984.

7. Kleinman A. The deligitimation and religitimation of local worlds. En: Good BJ, editor. Pain as human experience: An anthropological perspective. Berkeley: University of California Press; 1994.

8. Del Monaco R. Dolores de cabeza, síntomas y enfermedad: un abordaje social sobre la cons- trucción clínica de la migraña. Intersecciones en Antropología. 2012;13(2):501-511.

9. Margulies S, Barber N, Recoder ML. VIH-SIDA y "adherencia" al tratamiento: Enfoques y perspectivas. Antípoda Revista de Antropología y Arqueología. 2006;(3):281-300.

10. Pedraz MV. La construcción de una ética médico-deportiva de sujeción: el cuerpo preso de la vida saludable. Salud Pública de México [Internet]. 2007 [citado 15 mar 2012];49(1):71-78. Disponible en: http://www.scielo.org. mx/scielo.php?script = sci arttext\&pid $=$ S0036-36342007000100010.

11. Crawford R. Boundaries of the self and the unhealthy other: Reflections on health, culture and AIDS. Social Science and Medicine. 1994;38(10): 1347-1365.

12. Foucault M. El nacimiento de la clínica. Buenos Aires: Siglo Veintiuno Editores; 2008.

13. Conrad P. Medicalization and social control. Annual Review of Sociology [Internet]. 1992 [citado 15 mar 2012];18:209-232. Disponible en: http://www.jstor.org/stable/2083452.

14. Illich I. Medical Nemesis: The expropriation of health. New York: Pantheon; 1976.

15. Conrad P, Schneider JW. Desviance and medicalization: From badness to sickness. Ohio: Merril Publish; 1985. 
16. Epele M. Lógica causal y (auto)cuidado: Paradojas en el control médico del VIH-SIDA. Papeles de Trabajo: Centro Interdisciplinario de Ciencias Etnolingüísticas y Antropológico-Sociales. 1997;(6):87-93.

17. Foucault M. Historia de la sexualidad: El uso de los placeres. Buenos Aires: Siglo Veintiuno Editores; 2010.

18. Taylor SJ, Bogdan R. Introducción a los métodos cualitativos de investigación. Barcelona: Paidós; 1996.

19. Glasser B, Strauss A. The discover of grounded theory: Strategies for qualitative research. Chicago: Aldine; 1967.
20. Camargo KR. A Biomedicina. Physis: Revista de Saúde Coletiva [Internet]. 1997 [citado 12 mar 2012];7(1):45-68. Disponible en: http://www. scielo.br/scielo.php?script =sci_arttext\&pid =S010373311997000100003\&lng = es\&nrm = iso\&tlng = pt.

21. Bates M. Una perspectiva crítica de la enfermedad de la arteria coronaria y de la cirugía del By Pass Coronario. Cuadernos Médico Sociales. 1990;(54):51-67.

22. Pierret J. The illness experience: state of knowledge and perspectives for research. Sociology of Health and Illness. 2003;25(3):4-22.

23. Epele M. Scars, harm and pain. Journal of Ethnicity in Substance Abuse. 2001;1(1):47-69.

\section{FORMA DE CITAR}

Del Monaco R. Autocuidado, adherencia e incertidumbre: tratamientos biomédicos y experiencias de pacientes en el dolor crónico de la migraña. Salud Colectiva. 2013;9(1):65-78.

Recibido el 13 de julio de 2012

Versión final presentada el 17 de octubre de 2012

Aprobado 5 de diciembre de 2012 